\title{
Homogenize Coupled Stokes-Cahn-Hilliard System to Darcy's Law for Two-Phase Fluid Flow in Porous Medium by Volume Averaging
}

\author{
Jie Chen ${ }^{\mathrm{a}}$, Shuyu Sun ${ }^{\mathrm{a}, \mathrm{b}, *}$, Zhengkang $\mathrm{He}^{\mathrm{a}}$ \\ ${ }^{a}$ School of Mathematics and Statistics, Xi'an Jiaotong University, Xi'an, P.R.China 710049 \\ ${ }^{b}$ Computational Transport Phenomena Laboratory, Division of Physical Science and Engineering, King \\ Abdullah University of Science and Technology, Thuwal 23955-6900, Kingdom of Saudi Arabia
}

\begin{abstract}
A technique of local volume averaging is applied to obtain general equations which depict mass and momentum transport of incompressible two-phase flow in porous media. Starting from coupled Stokes-Cahn-Hilliard equations for incompressible twophase fluid flow, the averaging is performed without oversimplifying either the porous medium or the fluid mechanical relations. The resulting equations are Darcy's law for two-phase flow with medium parameters which could be evaluated by experiment.
\end{abstract}

Keywords: volume averaging, porous media, Stokes-Cahn-Hilliard equations, Darcy's law for two-phase flow

\section{Introduction}

The study of porous media flow is a delicate multiscale problem with many important applications such as the petroleum industry, fuels cells and agriculture $[15,2,32$, $25,22]$. On one hand, a full problem without any approximations is not computational tractable with the computational power so far. On the other hand, from an empirical perspective the consideration of the full multiscale problem is very challenging due to the difficulty of obtaining detailed information about the pore geometry and physical properties of the solid. These restrictions strongly call for systematic and reliable approximations which capture the essential physics and elementary dynamic characteristics of the full problem in an averaged sense. The widely used upscaling techniques can be divided into two main categories. One is the multiple scale expansion (or homogenization) technique $[3,8,16]$, and the other is volume averaging method

\footnotetext{
* Corresponding author

Email addresses: chenjiexjtu@mail.xjtu.edu.cn (Jie Chen),

shuyu. sun@kaust.edu.sa (Shuyu Sun), hzk2abc@163.com (Zhengkang He)

${ }^{1}$ The work is supported by the National Natural Science Foundation of China (No.11401467), China Postdoctoral Science Foundation (No. 2013M542334. and No. 2015T81012) and Natural Science Foundation of Shaanxi Province (No. 2015JQ1012).
} 
$[18,27,28,9]$. The former method has a rigorous mathematical verification under certain prerequisites. The latter one is more flexible and easy to implement.

Single phase flow in porous media is well understood in both Darcy's and pore scales. However, the modeling of two-phase flow in porous media are mainly based on empirical approaches. As the research of transport processes for two-phase flow in porous media advances to increasingly complex phenomena, the need becomes more acute for a unified, rigorous, and general methodology for the development of macroscopic transport equations from accepted first principles. Very few works have been done on this subject. In [20], Schmuck et al. derive a new effective macroscopic CahnHilliard equation in strongly heterogeneous domains and claim it is the first attempt of upscaling the Cahn-Hilliard equation in such domains. And then they derive effective macroscopic Stokes-Cahn-Hilliard equations for periodic immiscible two-phase flows in porous media under the assumption of periodic flow and a sufficiently large Péclet number in [21]. Daly and Roose [10] employ the multiple scale expansion technique to upscale the coupled Stokes-Cahn-Hilliard system to the Richards's equation. This method can capture the hysteresis in the water release curve and link the macroscopic properties of the porous media with the microscopic geometrical and material properties. Recently, Xu and Wang discuss the non-Darcy behavior of two-phase fluid flow in a single channel in [31].

In this paper, we employ the volume averaging technique on the coupled StokesCahn-Hilliard system to obtain general equations for two-phase fluid flow in a deforming porous medium, which is shown to reduce to Darcy's law for two-phase flow in the limit of slow flow. The rest of the paper is organized as follows. We introduce the extended volume averaging technique that will be utilized in this work in section 2. In section 3, the general equations for two-phase fluid flow in a deforming porous medium are derived from coupled Stokes-Cahn-Hilliard system by volume averaging procedure. The derived model is discussed with some numerical studies in section 4 . Conclusions are put in section 5 .

\section{An Extended Volume Averaging Technique for a Three-Phase System}

To develop averaged transport equations appropriate for incompressible two-phase fluid flow in porous media, one must be very careful to define clearly all the quantities that are being averaged and the volume over which the averaging is being performed. For the present analysis a three-phase system (Figure 1) will be considered in which $w$ denotes the wetting fluid phase, $o$ denotes the non-wetting fluid phase and $\beta$ denotes the solid phase. For the simplicity of illustration, the void volume occupied by fluid flow is denoted by $\alpha$.

The averaging process will lead to meaningful results provided the following length scale constraints are satisfied [27, 28, 9, 19,24].

$$
l_{\alpha}<<r_{0}<<L,
$$

where $l_{\alpha}$ is a microscopic characteristic length over which significant variations in the point quantities occur, $r_{0}$ is a characteristic length of the averaging volume, and $L$ is a macroscopic characteristic length. 
In this work it is assumed that the averaging volume is independent of time and of location in porous medium and has the proper characteristic length; the averaged equations obtained are independent of geometry of the averaging volume. The averaging volume is composed of both the $\alpha$ (void volume) and the $\beta$ phase such that

$$
V=V_{\alpha}(t)+V_{\beta}(t)
$$

where $V_{i}(t)$ is the volume of the $i$ phase in $V$ and is a function of time as well as space. Since the saturated porous medium are considered, the void volume are fully occupied by wetting and non-wetting fluid phases, i.e.

$$
V_{\alpha}(t)=V_{w}(t)+V_{o}(t) .
$$

A volume average of some quantity $\omega$ in the averaging volume is called superficial average and is defined by

$$
\left\langle\omega_{\alpha}\right\rangle=\frac{1}{V} \int_{V} \omega_{\alpha} \mathrm{d} V
$$

where $\omega_{\alpha}$ refers to the value of $\omega$ in the $\alpha$ phase and is defined to be zero in the $\beta$ phase. An average formed by integration over a specific phase is called an intrinsic phase average and is defined by

$$
\left\langle\omega_{\alpha}\right\rangle^{\alpha}=\frac{1}{V_{\alpha}} \int_{V_{\alpha}} \omega_{\alpha} \mathrm{d} V
$$

Note that with $\omega_{\alpha}$ equals to zero in the $\beta$ phase, the equality given in (2.2) can be written as

$$
\left\langle\omega_{\alpha}\right\rangle^{\alpha}=\frac{1}{V_{\alpha}} \int_{V} \omega_{\alpha} \mathrm{d} V
$$

If the fraction of the total volume occupied by the fluid phases (the $\alpha$ phase) is denoted by $\varepsilon_{\alpha}=V_{\alpha}(t) / V$, then

$$
\left\langle\omega_{\alpha}\right\rangle=\varepsilon_{\alpha}\left\langle\omega_{\alpha}\right\rangle^{\alpha}
$$

Analogous definitions apply to an intrinsic phase average taken over the $w, o, \beta$ phases respectively.

When a differential equation is averaged over a volume, terms arise which are averages of derivatives. These terms are related to the derivatives of averages by the following transport and averaging theorem:

Theorem 2.1. Transport theorem [29]

$$
\left\langle\frac{\partial \omega_{\alpha}}{\partial t}\right\rangle=\frac{\partial}{\partial t}\left\langle\omega_{\alpha}\right\rangle-\frac{1}{V} \int_{A_{\alpha \beta}} \omega_{\alpha} \mathbf{v}_{\alpha} \cdot \mathbf{n}_{\alpha} \mathrm{d} S .
$$


Averaging theorem [30]

$$
\left\langle\nabla \omega_{\alpha}\right\rangle=\nabla\left\langle\omega_{\alpha}\right\rangle+\frac{1}{V} \int_{A_{\alpha \beta}} \omega_{\alpha} \mathbf{n}_{\alpha} \mathrm{d} S .
$$

Modified averaging theorem [11]

$$
\left\langle\nabla \omega_{\alpha}\right\rangle=\varepsilon_{\alpha} \nabla\left\langle\omega_{\alpha}\right\rangle^{\alpha}+\frac{1}{V} \int_{A_{\alpha \beta}} \hat{\omega}_{\alpha} \mathbf{n}_{\alpha} \mathrm{d} S .
$$

Here $A_{\alpha \beta}$ is the area of the $\alpha-\beta$ interface in $V, \mathbf{v}_{\alpha}$ is the velocity of the $\alpha-\beta$ interfacial surface in $V, \mathbf{n}_{\alpha}$ is a unit normal vector along the $\alpha-\beta$ interface pointing outward from the $\alpha$ phase, and $\hat{\omega}_{\alpha}$ is the deviation of $\omega_{\alpha}$ from its intrinsic phase average defined by

$$
\hat{\omega}_{\alpha}=\omega_{\alpha}-\left\langle\omega_{\alpha}\right\rangle^{\alpha}
$$

at a point in the $\alpha$ phase.

The above theorem and definitions form a framework within which the equations describing transport in porous media can be derived. In the following sections, the coupled Stokes-Cahn-Hilliard equations will be averaged, and the conditions under which the averaged form reduces to Darcy's law for two-phase fluid flow will be enumerated.

\section{Equation Development}

\subsection{Coupled Stokes-Cahn-Hilliard equations}

The coupled Stokes-Cahn-Hilliard system for two-phase fluid flow reads as following:

$$
\begin{array}{r}
\nabla \cdot \boldsymbol{\sigma}+\mu \nabla \phi+\rho g_{e x t}=\nabla p, \\
\frac{\partial \phi}{\partial t}+\mathbf{u} \cdot \nabla \phi=M \Delta \mu, \\
\nabla \cdot \mathbf{u}=0,
\end{array}
$$

where $p$ is the pressure, $\boldsymbol{\sigma}=\nu\left(\nabla \mathbf{u}+\nabla \mathbf{u}^{T}\right)$ denotes the viscous part of the stress tensor, $\rho, \nu$ are the fluid mass density and viscosity. $\rho g_{\text {ext }}$ is the external body force density, and $M$ is the phenomenological mobility coefficient; $\mu=-N \Delta \phi-r \phi+u \phi^{3}$ is the chemical potential, and $\mu \nabla \phi$ is the capillary force; $N, r, u$ are the parameters that are related to the interface profile thickness $\xi=\sqrt{N / r}$, the interfacial tension $\gamma=2 \sqrt{2} r^{2} \xi / 3 u$, and the two homogeneous equilibrium phases $\phi_{ \pm}= \pm \sqrt{r / u}$.

According to [17], the velocity along the solid boundary satifies the generalized Navier boundary condition (GNBC),

$$
\beta u_{\tau}=-\nu\left(\partial_{\mathbf{n}} u_{\tau}+\partial_{\tau} u_{\mathbf{n}}\right)+L(\phi) \partial_{\tau} \phi,
$$

where $L(\phi)=N \partial_{\mathbf{n}} \phi+\partial \gamma_{\omega f}(\phi) / \partial \phi, \gamma_{\omega f}(\phi)=-\frac{1}{2} \gamma \cos \theta_{s} \sin \left(\frac{\pi}{2} \phi\right), \theta_{s}$ is the static contact angle, $u_{\mathbf{n}}$ and $u_{\tau}$ are the normal velocity and tangent velocity respectively, 
where $\mathbf{n}$ and $\tau$ are the unit normal and tangent vector of the slip boundary. In addition, a dynamic boundary condition is imposed on the phase field variable $\phi$ at the slip boundary,

$$
\frac{\partial \phi}{\partial t}+u_{\tau} \partial_{\tau} \phi=-\Pi(L(\phi))
$$

where $\Pi$ is a phenomenological parameter and should be positive. And also, the following non-penetration boundary conditions are used on solid boundaries.

$$
u_{\mathbf{n}}=\mathbf{u} \cdot \mathbf{n}=0, \quad \partial_{\mathbf{n}} \mu=0 .
$$

Following [17], we scale length by a reference length $L$, velocity by the reference velocity $V$, time by $L / V$ and pressure by $V / L$, and the following dimensionless equations are obtained.

$$
\begin{array}{r}
\nu \Delta \mathbf{u}+\mathcal{B} \mu \nabla \phi=\nabla p \\
\frac{\partial \phi}{\partial t}+\mathbf{u} \cdot \nabla \phi=\mathcal{L}_{d} \Delta \mu \\
\nabla \cdot \mathbf{u}=0 .
\end{array}
$$

Here $p$ is the pressure, $\mathbf{u}$ is the velocity, $\nu$ is the viscosity, $t$ is the time, $\phi$ is the phase field variable. $\mathcal{L}_{d}$ is the phenomenological mobility coefficient; $\mu$ is the chemical potential and has the expression that $\mu=-\epsilon \Delta \phi-\phi / \epsilon+\phi^{3} / \epsilon ; \mu \nabla \phi$ is the capillary force; $\epsilon$ is the ratio between dynamic interface thickness $\xi$ and characteristic length $L$. Notice that we have neglected external body force here, which can be easily incorporated into the above equations. The boundary conditions at solid walls are,

$$
\begin{array}{r}
\frac{u_{\tau}^{s l i p}}{\mathcal{L}_{s}}=-\nu\left(\partial_{\mathbf{n}} u_{\tau}+\partial_{\tau} u_{\mathbf{n}}\right)+\mathcal{B} L(\phi) \partial_{\tau} \phi, \\
\phi_{t}+u_{\tau} \partial_{\tau} \phi=-\mathcal{V}_{s} L(\phi), \\
u_{\mathbf{n}}=0, \\
\partial_{\mathbf{n}} \mu=0,
\end{array}
$$

where $L(\phi)=\epsilon \partial_{\mathbf{n}} \phi-\frac{\sqrt{2}}{6} \pi \cos \theta_{s} \cos \left(\frac{\pi}{2} \phi\right)$. Five dimensionless parameters appear in the above equations. They are (1) $\mathcal{L}_{d}=3 M \gamma / 2 \sqrt{2} V L^{2},(2) \mathcal{B}=3 \gamma / 2 \sqrt{2} V,(3) \mathcal{V}_{s}=$ $3 \gamma \Pi L / 2 \sqrt{2} V,(4) \mathcal{L}_{s}=1 / \beta(\phi) L$, which is the ratio of the slip length $l_{s}(\phi)=1 / \beta(\phi)$ to $L$. (5) $\epsilon$, which is the ration between interface thickness $\xi$ and characteristic length $L$.

Since the fluid flow in porous media usually transport slowly, a stationary Stokes equation (3.7) is used to couple with the Cahn-Hilliard equation (3.8) to depict the twophase flow in porous media. The incompressible flows are considered in this work, so the continuity equation reduces to (3.9). The GNBC (3.10-3.13) are used on the fluid-solid interface. The boundary condition (3.10) means the slip velocity on the fluid-solid interface is proportional to the tangential force acting on fluids. The first term on the righthand side of (3.10) is the tangential component of stress tensor and 
the second term is uncompensated Young stress [17]. Boundary condition (3.11) is the evolution equation of the phase function $\phi$ on the fluid-solid interface . (3.12) means the solid phase is impermeable. (3.13) means the diffusion along the normal direction on the fluid-solid interface is neglected, which ensures that the mass of wetting and non-wetting phases are conserved throughout the simulation.

Before we start the averaging process, the macroscopical variables are defined as follows:

Definition 3.1. The superficial average velocity for wetting fluid (w) phase and nonwetting fluid (o) phase are defined as

$$
\mathbf{u}_{w}=\left\langle\mathbf{u} \frac{1+\phi}{2}\right\rangle, \quad \mathbf{u}_{o}=\left\langle\mathbf{u} \frac{1-\phi}{2}\right\rangle
$$

The intrinsic average pressure for wetting fluid (w) phase and non-wetting fluid (o) phase are defined as

$$
p_{w}=\left\langle p \frac{1+\phi}{2}\right\rangle^{\alpha}, \quad p_{o}=\left\langle p \frac{1-\phi}{2}\right\rangle^{\alpha} .
$$

The saturation of wetting fluid $(w)$ phase and non-wetting fluid (o) phase are defined as

$$
S_{w}=\left\langle\frac{1+\phi}{2}\right\rangle^{\alpha}, \quad S_{o}=\left\langle\frac{1-\phi}{2}\right\rangle^{\alpha} .
$$

Remark 3.2. The physical meaning of saturation is the portion of phase volume in the void volume. For example, $S_{w}=V_{w} / V_{\alpha}$, so the above definition is natural. The use of the intrinsic average pressure results from the fact that $p_{w}$ and $p_{o}$ are the pressures that one might measure with a typical probe, or that one might be able to specify at a boundary. The use of the superficial average velocity follows from the convenient form of the continuity equation for incompressible flow.

\subsection{Upscale the continuity equation}

The superficial average of the continuity equation (3.9) for the $\alpha$ or fluid phase is

$$
\langle\nabla \cdot \mathbf{u}\rangle=0 \text {. }
$$

Applying (2.6), the averaging theorem, to the right-hand side term of the above equation yields

$$
\langle\nabla \cdot \mathbf{u}\rangle=\nabla \cdot\langle\mathbf{u}\rangle+\frac{1}{V} \int_{A_{\alpha} \beta} \mathbf{u} \cdot \mathbf{n}_{\alpha} \mathrm{d} S
$$

Applying the non-penetration boundary conditions (3.12) on the fluid-solid interface, we can get the averaged divergence free condition.

$$
\nabla \cdot\langle\mathbf{u}\rangle=0
$$




\subsection{Homogenization of the Cahn-Hilliard equation}

For Cahn-Hilliard equation, instead of using (3.8) directly, we average the following origin form of (3.8).

$$
\left\langle\frac{\partial \phi}{\partial t}\right\rangle+\langle\nabla \cdot \mathbf{u} \phi\rangle=\left\langle\mathcal{L}_{d} \Delta \mu\right\rangle .
$$

Application of the transport theorem (2.5) to the first term in this expression we get

$$
\begin{aligned}
& \left\langle\frac{\partial \phi}{\partial t}\right\rangle \\
= & \frac{\partial}{\partial t}\langle\phi\rangle-\frac{1}{V} \int_{A_{\alpha \beta}} \phi \mathbf{v}_{\alpha} \cdot \mathbf{n}_{\alpha} \mathrm{d} S \\
= & \frac{\partial}{\partial t}\langle\phi\rangle,
\end{aligned}
$$

where $\mathbf{v}_{\alpha}=0$, since the fluid-solid interface is static. Using the averaging theorem (2.6) to average the third term in equation (3.15), we have

$$
\begin{aligned}
& \langle M \Delta \mu\rangle \\
= & M\langle\nabla \cdot \nabla \mu\rangle \\
= & M \nabla \cdot\langle\nabla \mu\rangle+\frac{M}{V} \int_{A_{\alpha \beta}} \nabla \mu \cdot \mathbf{n}_{\alpha} \mathrm{d} S \\
= & M \nabla \cdot \nabla\langle\mu\rangle+\frac{M}{V} \nabla \cdot \int_{A_{\alpha \beta}} \mu \mathbf{n}_{\alpha} \mathrm{d} S+\frac{M}{V} \int_{A_{\alpha \beta}} \nabla \mu \cdot \mathbf{n}_{\alpha} \mathrm{d} S \\
= & M \nabla \cdot \nabla\langle\mu\rangle,
\end{aligned}
$$

where the assumption $\phi$ is sufficiently smooth (at least fourth order continuous), thus $\mu$ is sufficiently smooth, is used and the boundary condition (3.13) is applied on the fluid-solid interface.

For the wetting phase, the expression (3.16) can be rewritten as

$$
\begin{aligned}
& \frac{\partial}{\partial t}\langle\phi\rangle \\
= & 2 \varepsilon_{\alpha} \frac{\partial}{\partial t}\left\langle\frac{1+\phi}{2}\right\rangle^{\alpha} \\
= & 2 \varepsilon_{\alpha} \frac{\partial}{\partial t} S_{w} .
\end{aligned}
$$


The second term in (3.15) can be expressed as

$$
\begin{aligned}
& \langle\nabla \cdot(\mathbf{u} \phi)\rangle \\
= & \nabla \cdot\langle\mathbf{u} \phi\rangle \\
= & \nabla \cdot\langle\mathbf{u}+\mathbf{u} \phi\rangle \\
= & 2 \nabla \cdot\left\langle\mathbf{u} \frac{1+\phi}{2}\right\rangle \\
= & 2 \nabla \cdot \mathbf{u}_{w},
\end{aligned}
$$

where the macroscopic divergence free condition (3.14) is used.

Thus substituting expressions (3.17), (3.18) and (3.19) into equation (3.15), the macroscopic transport equation for wetting fluid phase is expressed as

$$
\varepsilon_{\alpha} \frac{\partial S_{w}}{\partial t}+\nabla \cdot \mathbf{u}_{w}=\frac{\mathcal{L}_{d}}{2} \Delta\langle\mu\rangle
$$

Analogously, the macroscopic transport equation for non-wetting fluid phase has the following expression:

$$
\varepsilon_{\alpha} \frac{\partial S_{o}}{\partial t}+\nabla \cdot \mathbf{u}_{o}=-\frac{\mathcal{L}_{d}}{2} \Delta\langle\mu\rangle
$$

\subsection{Homogenization of the Stokes equation}

The phase average of Stokes equation (3.7) for the fluid phase is

$$
\langle\nu \Delta \mathbf{u}\rangle+\langle\mathcal{B} \mu \nabla \phi\rangle=\langle\nabla p\rangle \text {. }
$$

Application of the averaging theorem (2.6) to the first term in (3.22) yields

$$
\begin{aligned}
& \langle\nu \Delta \mathbf{u}\rangle \\
= & \nu \nabla \cdot\langle\nabla \mathbf{u}\rangle+\frac{\nu}{V} \int_{A_{\alpha \beta}} \nabla \mathbf{u} \cdot \mathbf{n}_{\alpha} \mathrm{d} S \\
= & \nu \nabla \cdot\left(\nabla\langle\mathbf{u}\rangle+\frac{1}{V} \int_{A_{\alpha \beta}} \mathbf{u n}_{\alpha} \mathrm{d} S\right)+\frac{\nu}{V} \int_{A_{\alpha \beta}} \nabla \mathbf{u} \cdot \mathbf{n}_{\alpha} \mathrm{d} S \\
= & \frac{\nu}{V} \int_{A_{\alpha \beta}} \nabla \mathbf{u} \cdot \mathbf{n}_{\alpha} \mathrm{d} S
\end{aligned}
$$

where the divergence free equation (3.9) has been used and in [23], Slattery has shown analytically and numerous experimental investigations confirm that the term $\nu \Delta\langle\mathbf{u}\rangle$ can be considered negligible in slow flow.

The integrand of the dominant term

$$
\frac{\nu}{V} \int_{A_{\alpha \beta}} \nabla \mathbf{u} \cdot \mathbf{n}_{\alpha} \mathrm{d} S
$$

is the viscous stress of the solid on the fluid phase at a point on the surface, and the 
expression as a whole is the phase average viscous drag of the solid on the fluid. It is reasonable to assume that for a Newtonian fluid this drag depends on the difference between solid and fluid phase average velocities and that if macroscopically there is no relative motion, the net drag will be zero. Mathematically, this is expressed as

$$
\frac{\nu}{V} \int_{A_{\alpha \beta}} \nabla \mathbf{u} \cdot \mathbf{n}_{\alpha} \mathrm{d} S=\nu T\left(\left\langle\mathbf{u}_{\beta}\right\rangle-\langle\mathbf{u}\rangle\right)
$$

and

$$
T(\mathbf{0})=0
$$

where $\left\langle\mathbf{u}_{\beta}\right\rangle$ is averaged velocity in the solid phase, thus $\left\langle\mathbf{u}_{\beta}\right\rangle=0$. By analogy with a procedure used by [30] for the evaluation of the mass transfer tortuosity function, the integral $\mathrm{T}$ can be expanded in a Taylor series about the point $\left\langle\mathbf{u}_{\beta}\right\rangle-\langle\mathbf{u}\rangle=0$ to obtain

$$
T=a_{1}\left(\left\langle\mathbf{u}_{\beta}\right\rangle-\langle\mathbf{u}\rangle\right)+a_{2}:\left(\left\langle\mathbf{u}_{\beta}\right\rangle-\langle\mathbf{u}\rangle\right)\left(\left\langle\mathbf{u}_{\beta}\right\rangle-\langle\mathbf{u}\rangle\right)+\cdots
$$

where $a_{i}$ are the coefficients in the Taylor expansion with $a_{i}$ being $i+1$ th rank tensors. If the higher-order terms in this series are neglected and the expression for $T$ is substituted back into (3.24), the integral becomes

$$
\frac{\nu}{V} \int_{A_{\alpha \beta}} \nabla \mathbf{u} \cdot \mathbf{n}_{\alpha} \mathrm{d} S=\nu a_{1}\left(\left\langle\mathbf{u}_{\beta}\right\rangle-\langle\mathbf{u}\rangle\right)
$$

In the coupled Stokes-Cahn-Hilliard system, $\mu \nabla \phi$ is the capillary force density. Strictly speaking, it should be the gradient of capillary force. It has nonzero values only in the diffuse interface region and it always equals to zero in the bulk of phase. The interface thickness $\xi$ is a small quantity and in the sharp interface limit, i.e. $\xi \rightarrow 0$, the limit of the gradient function is a delta function. This explains why we usually call $\mu \nabla \phi$ capillary force in the coupled Stokes-Cahn-Hilliard system. Anyway, it is reasonable to assume the second term in (3.22) to be

$$
\langle\mu \nabla \phi\rangle=\nabla p_{c}
$$

where $p_{c}$ is the capillary pressure which equals to the pressure difference of two fluid phases, i.e., $p_{c}=p_{o}-p_{w}$.

Applying the modified averaging theorem (2.7) to the third term in (3.22), we get

$$
\langle\nabla p\rangle=\varepsilon_{\alpha} \nabla\langle p\rangle^{\alpha}+\frac{1}{V} \int_{A_{\alpha \beta}} \hat{p} \mathbf{n}_{\alpha} \mathrm{d} S .
$$

To complete the analysis, a constitutive relation for the remaining surface integral in (3.29) must be developed. If the function $p$ is independent of space, $\langle p\rangle^{\alpha}$ will be independent of space, and therefore $\nabla\langle p\rangle^{\alpha}$ as well as $\hat{p}$ will be zero. Alternatively one could say that if $\hat{p}$ is zero, $\nabla\langle p\rangle^{\alpha}$ is zero. For this case, the integral in (3.29) will be zero. Similarly $\hat{p}$ may be nonzero perturbations around values of $\langle p\rangle^{\alpha}$ such that $\nabla\langle p\rangle^{\alpha}$ still equals zero and $\hat{p}$ is also zero. These observations suggest that the surface integral 
is nonzero only to the extent that $\nabla\langle p\rangle^{\alpha}$ is nonzero. Thus one may express the integral as

$$
\frac{1}{V} \int_{A_{\alpha \beta}} \hat{p} \mathbf{n}_{\alpha} \mathrm{d} S=r\left(\nabla\langle p\rangle^{\alpha}\right)
$$

and

$$
r(\mathbf{0})=0
$$

where $r$ depends also on medium characteristics and possibly temperature.

A Taylor series expansion for $r$ around the point $r(\mathbf{0})$ yields

$$
\frac{1}{V} \int_{A_{\alpha \beta}} \hat{p} \mathbf{n}_{\alpha} \mathrm{d} S=b_{1} \nabla\langle p\rangle^{\alpha}+b_{2}: \nabla\langle p\rangle^{\alpha} \nabla\langle p\rangle^{\alpha}+\cdots
$$

where $b_{i}$ are the coefficients in the Taylor expansion with $b_{i}$ being $i+1$ th rank tensors. Insertion of the above relation together with (3.27), (3.28) and (3.29) into (3.22) yields

$$
\nu a_{1}\left(\left\langle\mathbf{u}_{\beta}\right\rangle-\langle\mathbf{u}\rangle\right)+\mathcal{B} \nabla p_{c}=\varepsilon_{\alpha} \nabla\langle p\rangle^{\alpha}+b_{1} \nabla\langle p\rangle^{\alpha} .
$$

Using the facts that $\langle\mathbf{u}\rangle=\mathbf{u}_{w}+\mathbf{u}_{o},\langle p\rangle^{\alpha}=p_{w}+p_{o}$ and $p_{c}=p_{o}-p_{w}$, the above equation can be rewritten as

$$
-\nu a_{1}\left(\mathbf{u}_{w}+\mathbf{u}_{o}\right)=\left(\varepsilon_{\alpha} \mathbf{I}+b_{1}+\mathcal{B} \mathbf{I}\right) \nabla p_{w}+\left(\varepsilon_{\alpha} \mathbf{I}+b_{1}-\mathcal{B} \mathbf{I}\right) \nabla p_{o},
$$

where $\mathbf{I}$ is the identity matrix. In porous media, it is natural to assume the flow of wetting/non-wetting phase is driven by the pressure difference of wetting/non-wetting phase. Thus we can split above equation into the following two equations

$$
\begin{aligned}
\mathbf{u}_{w} & =-\frac{\left(\varepsilon_{\alpha} \mathbf{I}+b_{1}+\mathcal{B} \mathbf{I}\right)\left(a_{1}\right)^{-1}}{\nu} \nabla p_{w}, \\
\mathbf{u}_{o} & =-\frac{\left(\varepsilon_{\alpha} \mathbf{I}+b_{1}-\mathcal{B} \mathbf{I}\right)\left(a_{1}\right)^{-1}}{\nu} \nabla p_{o} .
\end{aligned}
$$

Define the permeability as

$$
\mathbf{K}=\left(\varepsilon_{\alpha} \mathbf{I}+b_{1}\right)\left(a_{1}\right)^{-1},
$$

and define the relative permeability as

$$
\begin{aligned}
K_{r w} & =\left(\varepsilon_{\alpha} \mathbf{I}+b_{1}+\mathcal{B} \mathbf{I}\right)\left(\varepsilon_{\alpha} \mathbf{I}+b_{1}\right)^{-1}, \\
K_{r o} & =\left(\varepsilon_{\alpha} \mathbf{I}+b_{1}-\mathcal{B} \mathbf{I}\right)\left(\varepsilon_{\alpha} \mathbf{I}+b_{1}\right)^{-1} .
\end{aligned}
$$


Then equations (3.35) can be rewritten as

$$
\begin{aligned}
\mathbf{u}_{w} & =-\frac{K_{r w}}{\nu} \mathbf{K} \nabla p_{w} \\
\mathbf{u}_{o} & =-\frac{K_{r o}}{\nu} \mathbf{K} \nabla p_{o}
\end{aligned}
$$

which are the Darcy's law for two-phase incompressible fluid flow in porous media.

\section{Discussion on Derived Model for Two-phase Flow in Porous media}

Summarize the equations derived in section 3, we get the following system for two-phase incompressible flow in porous media.

$$
\begin{aligned}
\varepsilon_{\alpha} \frac{\partial S_{w}}{\partial t}+\nabla \cdot \mathbf{u}_{w} & =\frac{\mathcal{L}_{d}}{2} \Delta\langle\mu\rangle \\
\varepsilon_{\alpha} \frac{\partial S_{o}}{\partial t}+\nabla \cdot \mathbf{u}_{o} & =-\frac{\mathcal{L}_{d}}{2} \Delta\langle\mu\rangle, \\
\mathbf{u}_{\gamma} & =-\frac{K_{r \gamma}}{\nu} \mathbf{K} \nabla p_{\gamma}, \quad \gamma=w, o \\
S_{w}+S_{o} & =1, \\
p_{c} & =p_{o}-p_{w} .
\end{aligned}
$$

(4.1) and (4.2) are the mass balance equations for each of the fluid phases. The volumetric velocity $\mathbf{u}_{\alpha}$ is governed by the Darcy's law (4.3). The constraint for the saturations is (4.4) and the two pressures are related by a given capillary pressure function (4.5).

Comparing to the empirical model of two-phase flow adopted in oil industry [7],

$$
\begin{aligned}
\varepsilon_{\alpha} \frac{\partial S_{\gamma}}{\partial t}+\nabla \cdot \mathbf{u}_{\gamma} & =0, \quad \gamma=w, o, \\
\mathbf{u}_{\gamma} & =-\frac{K_{r \gamma}}{\nu} \mathbf{K} \nabla p_{\gamma}, \quad \gamma=w, o, \\
S_{w}+S_{o} & =1 \\
p_{c}\left(S_{w}\right) & =p_{o}-p_{w},
\end{aligned}
$$

the differences lie on two aspects. First, the diffusion term $\pm \frac{\mathcal{L}_{d}}{2} \Delta\langle\mu\rangle$ in mass balance equations (4.1) and (4.2) are omitted by the traditional model. Second, (3.28) is assumed by us from the physical concept, where $p_{c}$ is a function of $S_{w}$ in (4.6). We will discuss these two aspects with numerical examples in the following.

\subsection{Deducing Richards' equation from the derived model}

In this subsection, the Richards's equation which governs the flow of liquids in unsaturated porous mediums will be deduced from the homogenized equations we have derived. 
We choose the saturation of wetting phase and the pressure of non-wetting phase as main variables. Then we introduce the phase mobility functions

$$
\lambda_{\gamma}\left(\mathbf{x}, S_{w}\right)=\frac{K_{r \gamma}}{\nu}, \quad \gamma=w, o,
$$

and the total mobility

$$
\lambda\left(\mathbf{x}, S_{w}\right)=\lambda_{w}+\lambda_{o}
$$

The fractional flow functions are defined by

$$
f_{\gamma}\left(\mathbf{x}, S_{w}\right)=\frac{\lambda_{\gamma}}{\lambda}, \quad \gamma=w, o .
$$

Notice the fact

$$
\langle\mathbf{u}\rangle=\mathbf{u}_{w}+\mathbf{u}_{o},
$$

where $\langle\mathbf{u}\rangle$ is the total velocity. From equation (3.34), we have

$$
\mathbf{u}_{w}+\mathbf{u}_{o}=-\frac{K_{r w}}{\nu} \mathbf{K} \nabla p_{w}-\frac{K_{r o}}{\nu} \mathbf{K} \nabla p_{o} .
$$

Equation (4.10) can be reformulated as

$$
\langle\mathbf{u}\rangle=-\mathbf{K}\left(\lambda\left(S_{w}\right) \nabla p_{o}-\lambda_{w}\left(S_{w}\right) \nabla p_{c}\right) .
$$

Apply (4.5) and (4.11) to (4.3) with $\gamma=w$ to have the expression of wetting phase velocity

$$
\begin{aligned}
\mathbf{u}_{w} & =-K \lambda_{w} \nabla p_{w} \\
& =K \lambda_{w} \nabla p_{c}-K \lambda f_{w} \nabla p_{o} \\
& =K\left(\lambda_{w}+\lambda_{o}\right) f_{w} \nabla p_{c}-K \lambda f_{w} \nabla p_{o} \\
& =f_{w}\langle\mathbf{u}\rangle+K \lambda_{o} f_{w} \nabla p_{c} .
\end{aligned}
$$

Substituting (4.12) into (4.1), we have

$$
\varepsilon_{\alpha} \frac{\partial S_{w}}{\partial t}+\nabla \cdot\left\{K \lambda_{o} f_{w} \nabla p_{c}+f_{w}\langle\mathbf{u}\rangle\right\}=\frac{\mathcal{L}_{d}}{2} \Delta\langle\mu\rangle .
$$

So far, in the models used for simulating two-phase flows in porous mediums, the capillary pressure is usually a function of saturation. For instance, in Brooks-Corey model $[4,12]$,

$$
p_{c}=p_{d}\left(\frac{S_{w}-S_{r w}}{1-S_{r o}-S_{r w}}\right)^{\frac{1}{\zeta}},
$$

where $p_{d}$ is a threshold pressure; $\zeta$ is the parameter associated with pore size distribu- 
tion; $S_{r w}$ and $S_{r o}$ are residual saturation of wetting and non-wetting phase respectively. In such case, using $\mathbf{u}_{w}=K \lambda_{w} \nabla p_{c}-K \lambda f_{w} \nabla p_{o}$, equation (4.13) can be rewritten as

$$
\varepsilon_{\alpha} \frac{\partial S_{w}}{\partial t}+\nabla \cdot\left\{K \lambda_{w} \frac{\mathrm{d} p_{c}}{\mathrm{~d} S_{w}} \nabla S_{w}-K \lambda_{w} \nabla p_{o}\right\}=\frac{\mathcal{L}_{d}}{2} \Delta\langle\mu\rangle .
$$

We have reached the mixed form of Richards' equation (4.15). The only difference is there is a term $\frac{\mathcal{L}_{d}}{2} \Delta\langle\mu\rangle$ on the righthand side of equation (4.15) and it will be discussed with numerical examples in next subsection.

\subsection{Numerical examples}

\subsubsection{Example 1: coalescence of two interacting bubbles}

Here we simulate the coalescence of two interacting bubbles. The computational domain is $[x, y]=[0,1.0] \times[0,1.0]$ and the mesh size of the triangular mesh is $h=0.02$. The time step is $d t=0.1 h=0.002$. The coupled Stokes-Cahn-Hilliard equations (3.7-3.9) are simulated with finite element methods [1, 5, 6, 13, 14]. In detail, The Stokes equation (3.7) is solved by P2-P1 finite elements and the Cahn-Hilliard equation (3.8) is solved by a convex splitting technique. The boundary conditions (3.10-3.13) are assigned on upper and lower boundaries and periodic boundary conditions are used on left and right boundaries, so that the computational domain can be considered as a representative elementary volume (REV) in a channel with infinite length in porous media. In Figure 2, the snapshots of phase variable $\phi$ are shown at eight different times.

We then calculate the averaged chemical potential $\langle\mu\rangle$ and averaged gradient of capillary force $\langle\mu \nabla \phi\rangle$ in this REV, which are shown in Figure 3 and 4, respectively. When the system approaches the equilibrium state, $\langle\mu\rangle$ remains a constant and $\langle\mu \nabla \phi\rangle$ converges to zero. In the field of fluid mechanics in porous medium, a quasi-static assumption is usually used, which means the system is considered as in the equilibrium state at every time step. In macro-scale, this REV can be seen as a single element. In this example, since $\langle\mu\rangle$ remains a constant, thus $\pm \frac{\mathcal{L}_{d}}{2} \Delta\langle\mu\rangle$ reduce to zero in macroscale. By definition, $S_{w}$ remains a constant in this REV. In the traditional model, $p_{c}$ is a function of $S_{w}$, so $p_{c}$ is a constant and $\nabla p_{c}$ is zero. This coincides with the numerical results shown in Figure 4 when a quasi-static assumption is made.

\subsubsection{Example 2: a droplet on the solid surface}

Example 2 simulates a droplet on the solid surface. The computational domain is $[x, y]=[0,1.0] \times[0,1.0]$ and the mesh size of the triangular mesh is $h=0.02$. The time step is $d t=0.1 h=0.002$. The boundary conditions (3.10-3.13) are assigned on upper and lower boundaries and periodic boundary conditions are used on left and right boundaries. In Figure 5, the snapshots of phase variable $\phi$ are shown at eight different times. Since the static contact angle is set to be $\frac{2 \pi}{3}$, the droplet is deformed under the surface tension until the contact angle reaches the static contact angle.

Due to the boundary conditions, the computational domain can be considered as a REV in a channel with infinite length in porous media. We then calculate the averaged chemical potential $\langle\mu\rangle$ and averaged gradient of capillary force $\langle\mu \nabla \phi\rangle$ in this REV, which are shown in Figure 6 and 7, respectively. $\langle\mu\rangle$ approaches a constant when the 
system approaches the equilibrium state. However, $\langle\mu \nabla \phi\rangle$ doesn't converges to zero in this case. That is to say $\nabla p_{c}$ is not zero although $S_{w}$ is a constant. When there a contact line (contact point in 2D), the traditional capillary pressure model such as (4.14) is no longer strictly valid. This illustrates that the capillary pressure is a function of not only saturation of wetting phase but also other factors. As a consequence, we recommend Equation (4.13) to be the general governing equation for two-phase flow in porous media instead of the traditional Richards' equation (4.15).

\subsubsection{Example 3: Two-phase fluid flows passing through the Taylor brush configura- tion}

Example 3 simulates two-phase fluid flows pass through the Taylor Brush Configuration [26]. The computational domain is shown in Figure 8. The mesh size of the triangular mesh is $h=0.02$. The time step is $d t=0.1 h=0.002$. The static contact angle is $\frac{2 \pi}{3}$. A parabolic velocity is imposed on the left boundary and the natural outflow boundary condition is imposed on the right boundary. The boundary conditions (3.10-3.13) are assigned on all the other boundaries, i.e. GNBC is imposed on all the fluid-solid interface. The computational domain is divided into 32 elementary volumes which is also shown in Figure 8. This example can be consider as two-phase fluid flows passing through a coupled free flow region and porous media region. Similar cases can be found in fractured or shale reservoirs, industry filters and so on.

Different from the previous two examples, there is no equilibrium state of this example. The snapshots of phase variable $\phi$ at eight different times are shown in Figure 9. We calculate the averaged chemical potential $\langle\mu\rangle$ on every elementary volume and averaged chemical potential $\langle\mu\rangle$ on elementary volume 7, 15, 19, 27 are shown in Figure 10. We can see that $\langle\mu\rangle$ varies a lot near the fluid-fluid interface. Then $\Delta\langle\mu\rangle$ on elementary volume $i$ is calculated by a central difference scheme

$$
\begin{array}{r}
\Delta\langle\mu\rangle_{i}=\frac{\langle\mu\rangle_{i-4}-2\langle\mu\rangle_{i}+\langle\mu\rangle_{i+4}}{(\delta x)^{2}}+\frac{\langle\mu\rangle_{i-1}-2\langle\mu\rangle_{i}+\langle\mu\rangle_{i+1}}{(\delta y)^{2}}, \\
i=6,7,10,11,14,15,18,19,22,23,26,27,
\end{array}
$$

where $\delta x=\delta y=0.25 . \Delta\langle\mu\rangle$ on elementary volume 7, 15, 19, 27 are shown in Figure 11. It is obvious that when two-phase fluid flows pass through the interface between the free flow region and the porous media region, the term $\Delta\langle\mu\rangle$ can't be omitted.

\section{Conclusions}

We derive a model for two-phase incompressible flow in porous media by upscaling the Stokes-Cahn-Hilliard system with local volume averaging technique. A comparison between the derived model and the commonly used empirical model is made and the differences are discussed with a numerical example. The derived model reduces to the traditional model under the quasi-static assumption. However, there are some situations that the diffusion term $\pm \frac{\mathcal{L}_{d}}{2} \Delta\langle\mu\rangle$ in mass balance equations are not negligible, such as two-phase flow passing through the interface between matrix and fracture in a fractured or shale reservoir. 


\section{References}

[1] Bao K., Shi Y., Sun S. and Wang X., A finite element method for the numerical solution of the coupled Cahn-Hilliard and Navier-Stokes system for moving contact line problems, Journal of Computational Physics, vol. 231, no. 24, pp. 8083-8099, 2012.

[2] Benet J-C., Ramirez-Martinez A., Ouedraogo F. and Cherblanc F., Measurement of the Chemical Potential of a Liquid in Porous Media, Journal of Porous media, vol. textbf15, no. 11, pp. 1019-1029, 2012.

[3] Bensoussan A., Lions J. L., and Papanicolaou G., Analysis for Periodic Structures. North-Holland Publishing Company, North-Holland, Amsterdam, 1978.

[4] Brooks R.H. and Corey T., Hydraulic Properties of Porous Media, in: Hydrol. Pap., vol. 3, 1964.

[5] Chen J., Sun S. and Wang X., A numericalmethod for amodel of two-phase flow in a coupled free flow and porous media system, Journal of Computational Physics, vol. 268, pp. 1-16, 2014.

[6] Chen J., Sun S. and Chen Z., Coupling Two-Phase Fluid Flow with Two-Phase Darcy Flow in Anisotropic Porous Media, Advances in Mechanical Engineering, vol. 6, pp. 871021, 2014.

[7] Chen Z., Huan G. and Ma Y., Computational methods for multiphase flows in porous media, Siam, 2006.

[8] Cioranescu D. and Donato P., An Introduction to Homogenization, volume 17 of Oxford Lecture Series in Mathematics and its Applications. Oxford University Press, New York, 1999.

[9] Dagan G., The generalization of Darcy's law for nonuniform flows, Water Resour. Res., vol. 15, pp. 1-7, 1979.

[10] Daly K. R. and Roose T., Homogenisation of two fluid flow in porous media. Proc. R. Soc. A, vol. 471, pp. 20140564, 2015.

[11] Gray W. G., A derivation of the equations for multi-phase transport, Chem. Eng. Sci., vol. 30, pp. 229-233, 1975.

[12] Hou J., Chen J., Sun S. and Chen Z., Adaptive mixed-hybrid and penalty discontinuous Galerkin method for two-phase flow in heterogeneous media, Journal of Computational and Applied Mathematics, vol. 307, pp. 262-283, 2016.

[13] Kou J., Sun S. and Wang X., Efficient numerical methods for simulating surface tension of multi-component mixtures with the gradient theory of fluid interfaces, Computer Methods in Applied Mechanics and Engineering, vol. 292, pp. 92-106, 2015. 
[14] Kou J., Sun S., Numerical Methods for a Multicomponent Two-Phase Interface Model with Geometric Mean Influence Parameters, SIAM Journal on Scientific Computing, vol. textbf37, no. 4, pp. B543-B569, 2015.

[15] Manafian J., Lakestani M. and Bekir A., Application of a New Analytical Method for the Richards Equation, Based on the Brooks and Corey Model, Journal of Porous media, xol. 19, no. 11, pp. 975-991, 2016.

[16] Pavliotis G. A. and Stuart A. M., Multiscale Methods, volume 53 of Texts in Applied Mathematics. Springer, New York, 2008.

[17] Qian T., Wang X. P. and Sheng P., Molecular scale contact line hydrodynamics of immiscible flows, Phys. Rew. E, vol. 68, pp. 016306, 2003.

[18] Quintard M. and Whitaker S., Transport in ordered and disordered porous media: volume-averaged equations, closure problems, and comparison with experiment. Chem. Engng Sci., vol. 48, no. 14, pp. 2537-2564, 1993.

[19] Sanchez-Palencia E., Non-homogeneous Media and Vibration Theory, Lecture Notes in Physics 127, Springer-Verlag, New York, 1980.

[20] Schmuck M., Pradas M., Pavliotis G. A. and Kalliadasis S., Upscaled phase-field models for interfacial dynamics in strongly heterogeneous domains. Proceedings of the Royal Society of London A: Mathematical, Physical and Engineering Sciences, The Royal Society, vol. 468, no. 2147, pp. 3705-3724, 2012.

[21] Schmuck M., Pavliotis G. A. and Kalliadasis S., Effective macroscopic StokesCahn-Hilliard equations for periodic immiscible flows in porous media. Proceedings of the European Conference on Complex Systems 2012, Springer International Publishing, pp. 1005-1010, 2013.

[22] Shi Y., Yang Z. and Yang D., Determination of Non-Darcy Flow Behavior in a Tight Formation, Journal of Porous media, vol. 19, no. 8, pp. 687-700, 2016.

[23] Slattery J. C., Momentum, Energy, and Mass Transfer in Continua, McGraw-Hill, New York, 1972.

[24] Tartar L., Incompressible fluid flow in a porous medium: Convergence of the homogenization process, Appendix in Lecture Notes in Physics 127, Springer-Verlag, New York, 1980.

[25] Tayari A., Bouadba R. and Magherbi M., Irreversibility Analysis in Mixed Convection Flow in a Porous Channel with Viscous Dissipation, Journal of Porous media, vol. 19, no. 10, pp. 853-870, 2016.

[26] Taylor G. I., A model for the boundary condition of a porous material. Part 1, J. Fluid Mech., vol. textbf49, pp. 319-326, 1971.

[27] Whitaker S., Flow in porous media I: A theoretical derivation of Darcy's law. Transport Porous Med., vol. 1, pp. 3-25, 1986. 
[28] Whitaker S., Advances in the theory of fluid motion in porous media, Ind. Eng. Chem. vol 12, pp. 14-28, 1969.

[29] Whitaker S., The transporte quationsf or multi-phase systems, Chem. Eng. Sci., vol. 28, pp. 139-147, 1973.

[30] Whitaker S., Diffusion and dispersion in porous media, AIChE J., vol. 13, pp. 420-427, 1967.

[31] Xu X. and Wang X., Non-Darcy behavior of two-phase channel flow. Physical Review E, vol. 90, no. 2, pp. 023010, 2014.

[32] Zhang R. and Wu Y., Sequentially Coupled Model for Multiphase Flow, Mean Stress, and Reactive Solute Transport with Kinetic Chemical Reactions: Applications in CO2 Geological Sequestration, Journal of Porous media, vol. 19, no. 11, pp. 1001-1021, 2016.

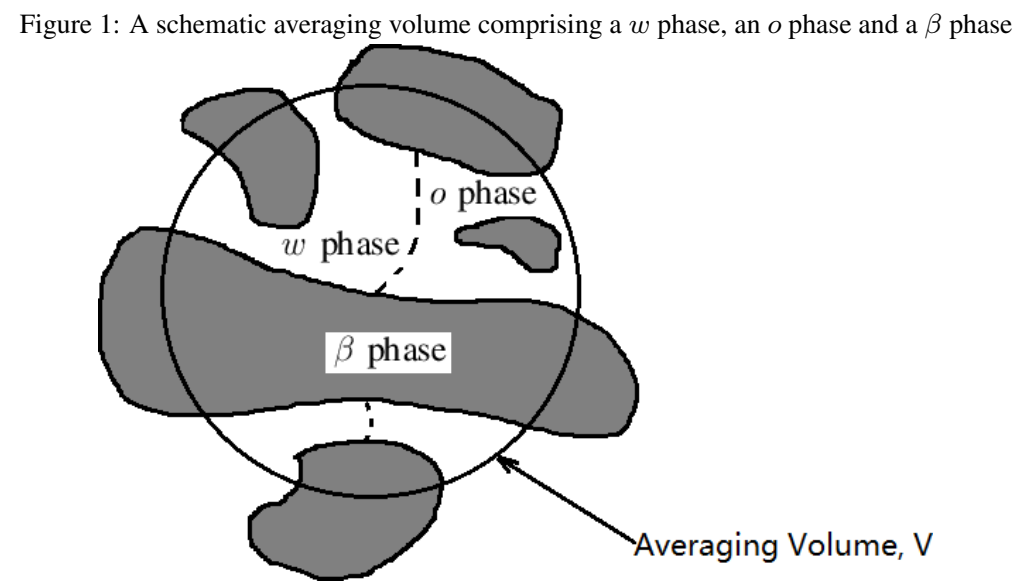


Figure 2: Snapshots of $\phi$ calculated in the computational domain $[0,1.0] \times[0,1.0]$ by time step $d t=0.1 h$ at time $t=0.0040,0.0200,0.0400,0.0600,0.0800,0.1000,0.1400,1.0000$.
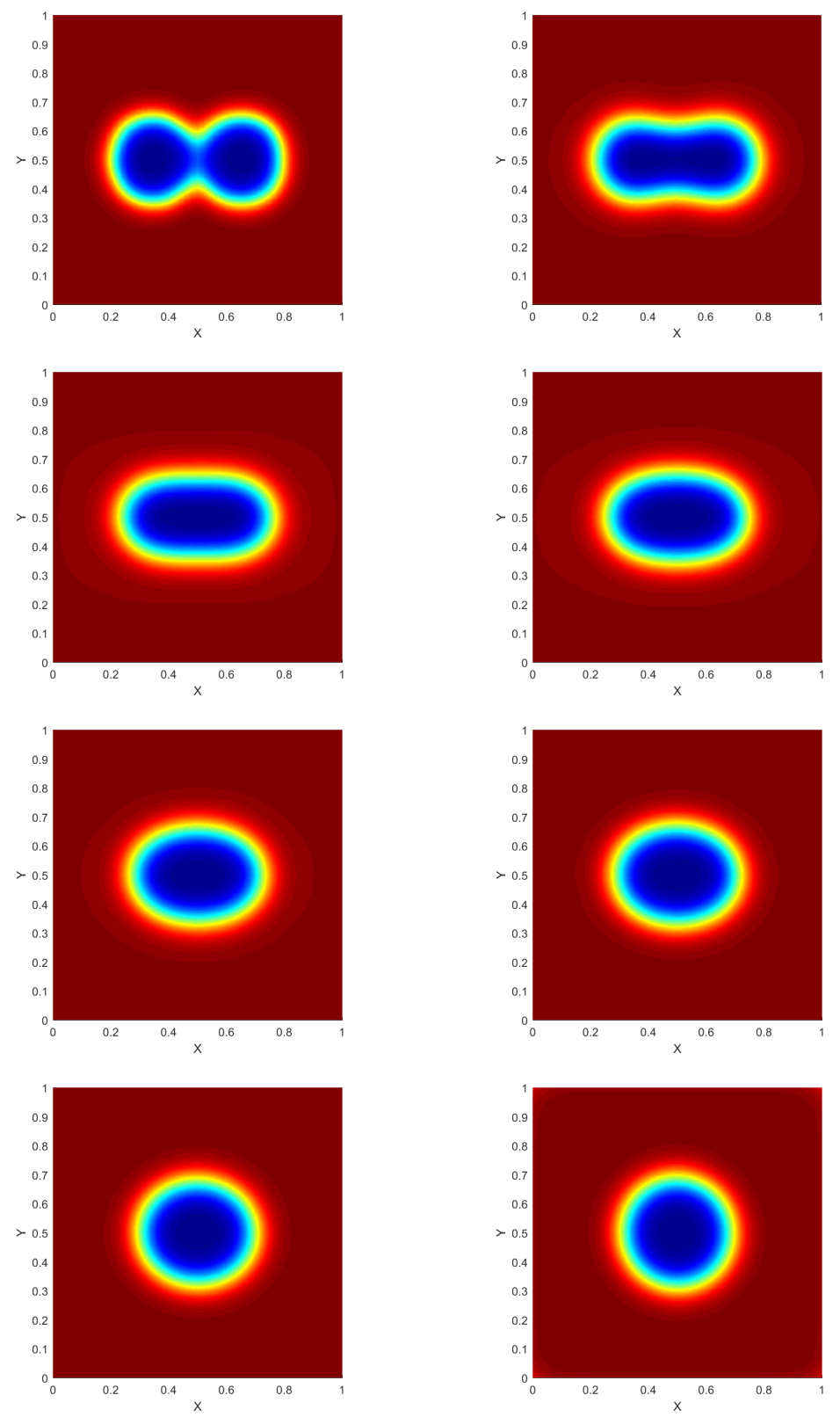
Figure 3: Averaged chemical potential

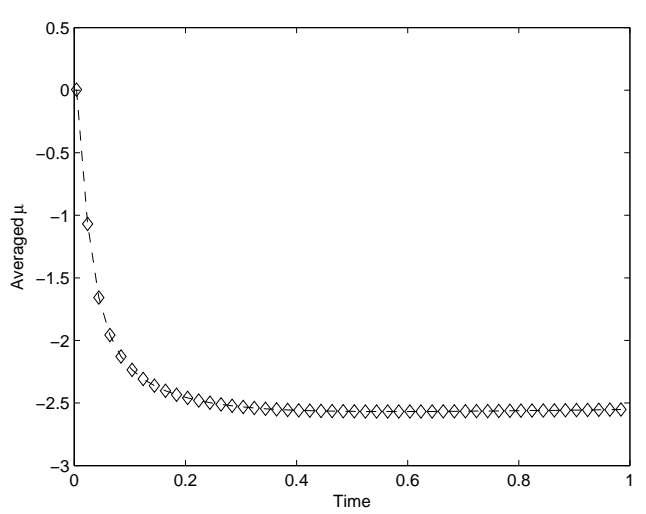

Figure 4: Averaged partial derivatives of capillary force

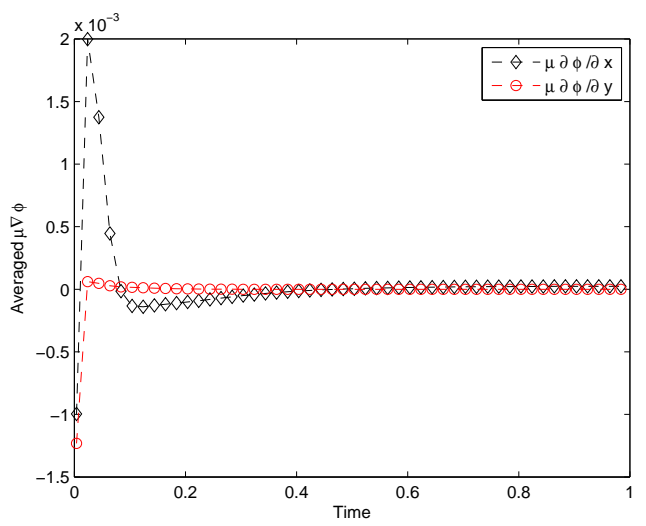


Figure 5: Snapshots of $\phi$ calculated in the computational domain $[0,1.0] \times[0,1.0]$ by time step $d t=0.1 \mathrm{~h}$ at time $t=0.0040,0.0200,0.0400,0.0600,0.0800,0.1000,0.1400,1.0000$.
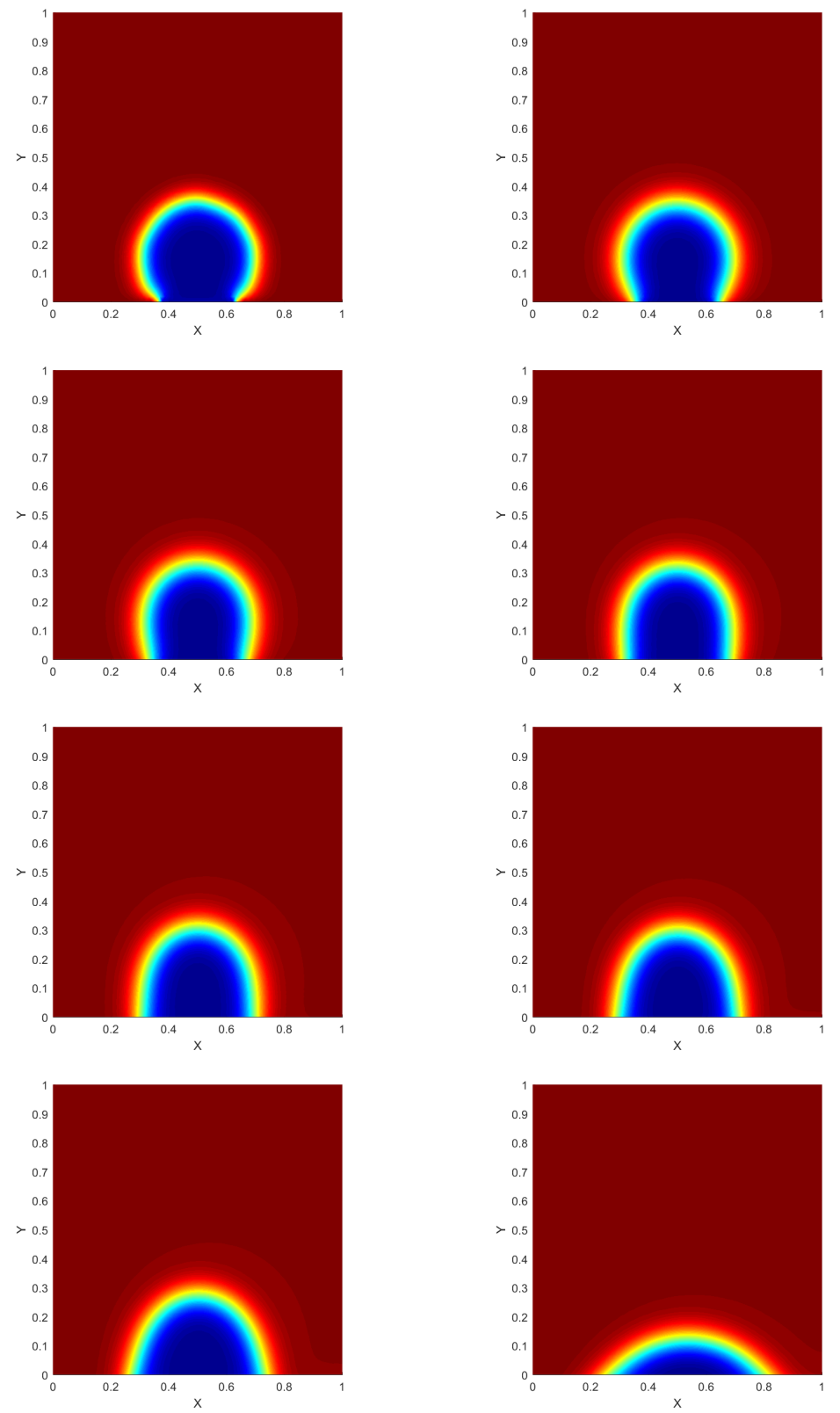
Figure 6: Averaged chemical potential

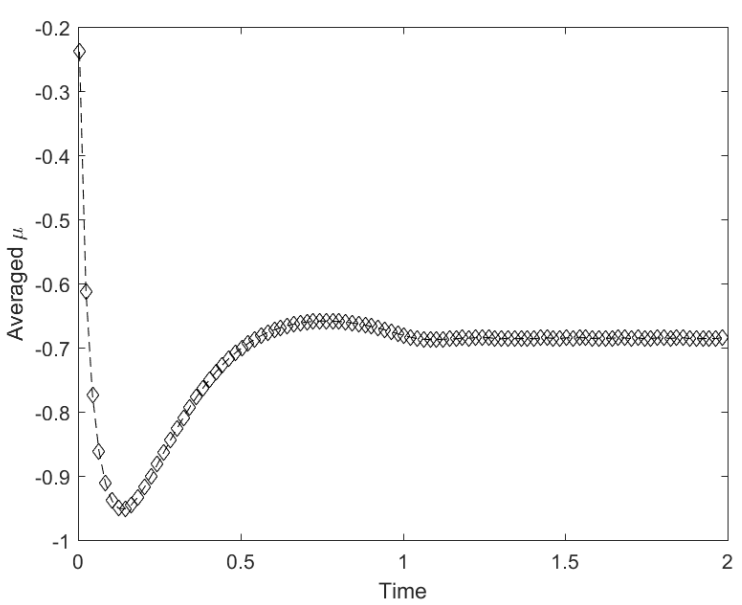

Figure 7: Averaged partial derivatives of capillary force

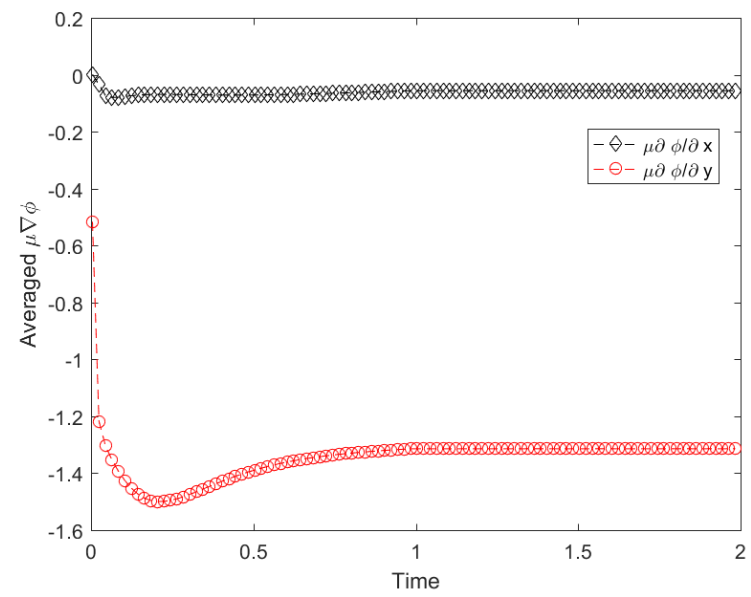


Figure 8: The computational domain of example 3: Taylor brush configuration. The triangular mesh is illustrated in green color. The whole computational domain is divided into 32 elementary volumes and labelled with red numbers.

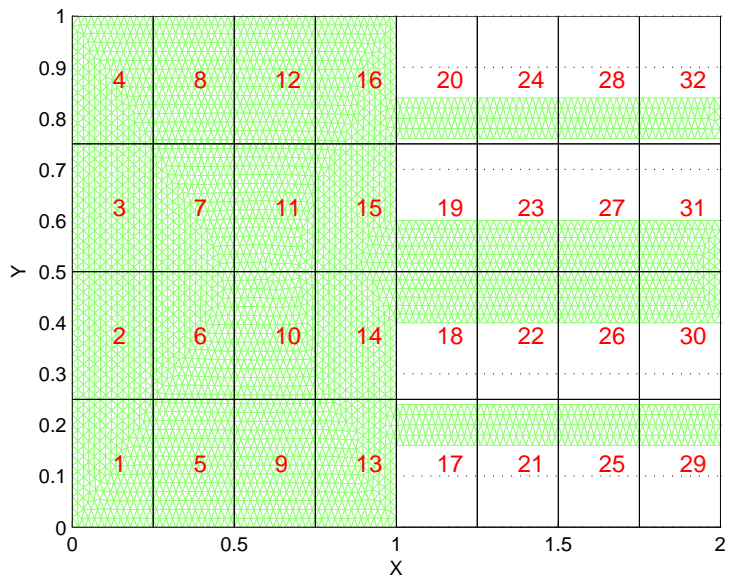


Figure 9: Snapshots of $\phi$ calculated in the Taylor brush configuration by time step $d t=0.1 \mathrm{~h}$ at time $t=0.0040,0.2000,0.3000,0.4000,0.5000,0.6000,0.7000,0.8000$.
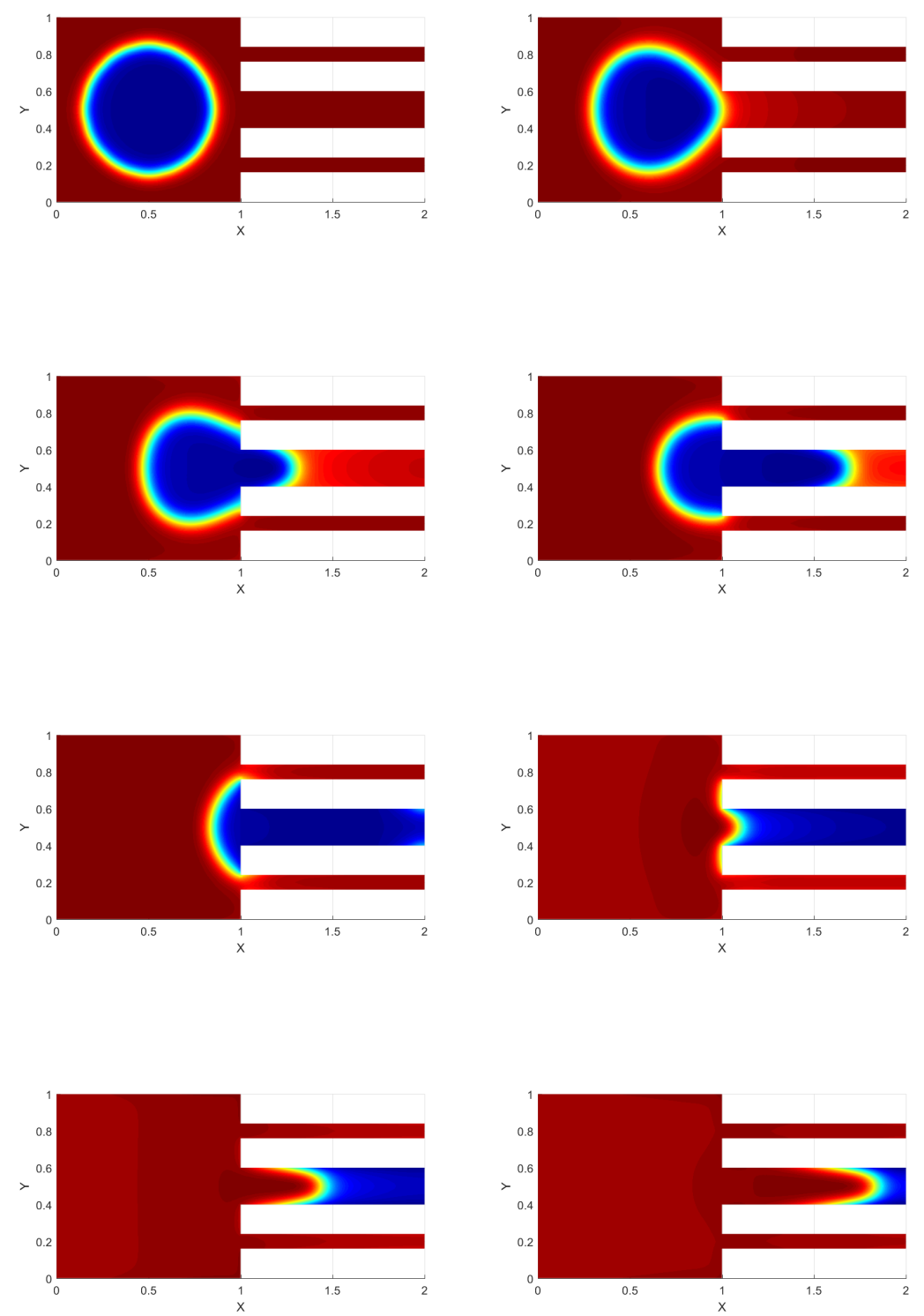
Figure 10: Averaged chemical potential on elementary volume 7 (up left), 15 (up right), 19 (down left), 27 (down right).
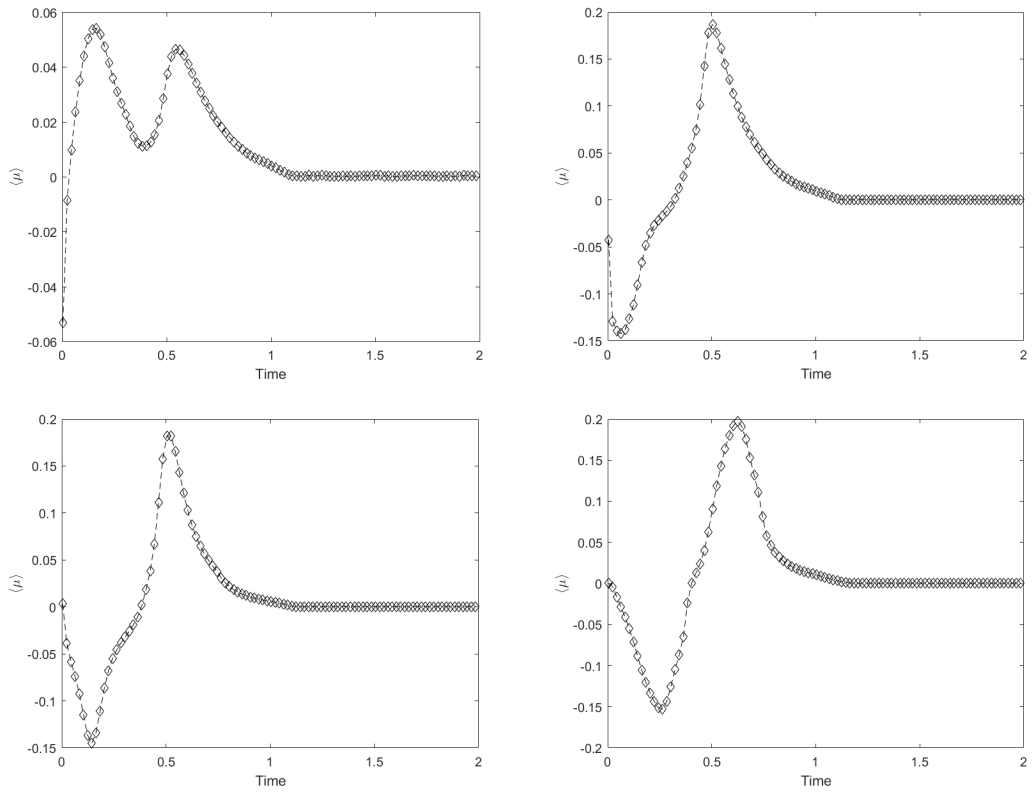

Figure 11: $\Delta\langle\mu\rangle$ on elementary volume 7 (up left), 15 (up right), 19 (down left), 27 (down right).
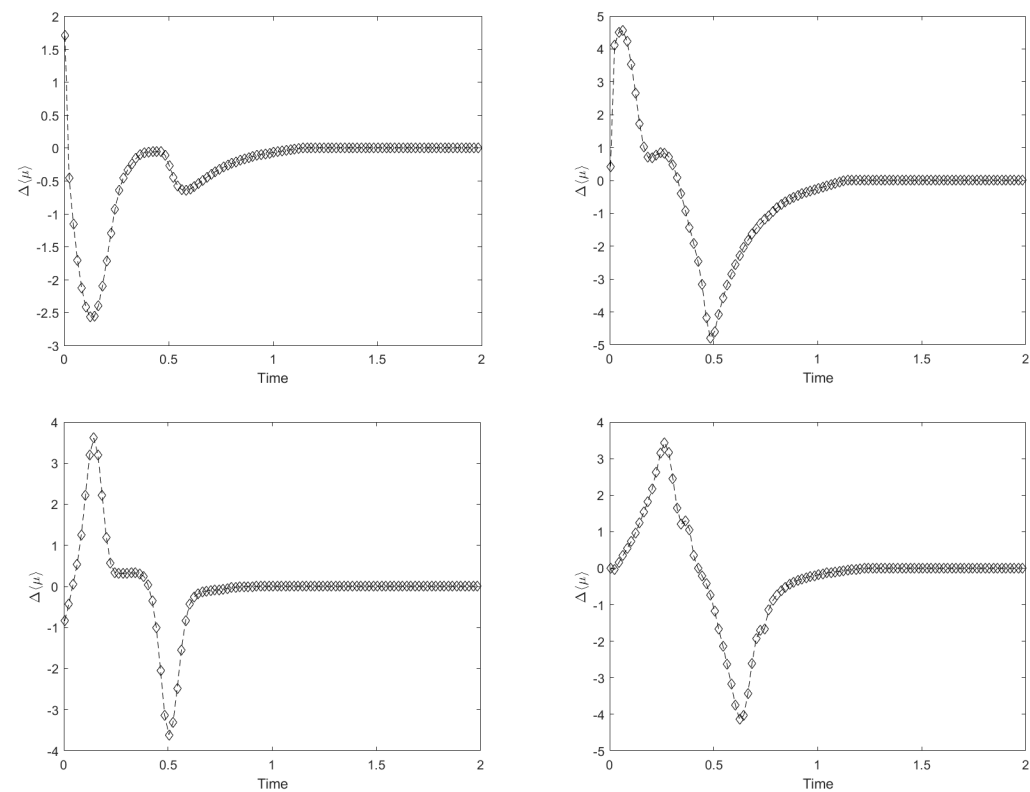